\title{
SALUD PÚBLICA DESDE LA PERSPECTIVA DE GÉNERO: HITOS E INNOVACIÓN
}

\author{
MARÍA TERESA Ruíz CANTERO \\ Universidad de Alicante
}

\section{Introducción}

Los estudios sobre la salud pública en España han proliferado en las últimas décadas, poniendo algunos, de ellos, de manifiesto que las mujeres, la población de menor nivel socioeconómico o las áreas con mayor privación material presentan unos indicadores de salud que denotan una peor situación. De ahí el interés que tiene para la salud pública el comprender y aplicar la perspectiva y el análisis de género. El elemento común que comparten el enfoque de la Salud Pública y la perspectiva de género es su consideración SOCIAL, lo que permite que el ANALISIS DE GÉNERO pueda tener lugar con relación a problemas y situaciones de salud de las mujeres y los hombres.

La salud de las mujeres y los hombres es diferente pero también desigual: diferente porque existen factores de tipo biológico y fisiológico que tienen distintas implicaciones en su salud; desigual porque hay factores sociales que establecen diferencias evitables, y por ello injustas, en la salud de ambos sexos.

Es en este último concepto en el que se centra el presente número de la revista feminismos, cuyas/os autoras y autores proceden de diferentes instituciones de Andalucía, Aragón, Cataluña, Comunidad Valenciana, Madrid y País Vasco, e internacionales, como Colombia y Suecia. Son las Universidades los centros de trabajo de las/los autoras/es más frecuentes (Universidad de: Antioquia, Nacional de Colombia, País Vasco, Rovira i Virgili, Sevilla, Umeå, Zaragoza y Alicante). También, participan profesionales de la Agencia de Salud Pública de Barcelona, Centro de Análisis y Programas Sanitarios de Barcelona, Conselleria de Sanidad de la Comunidad Valenciana, Escuela Andaluza 
de Salud Pública, Escuela Nacional de Sanidad, Hospital de Madrid y Secretaría del Plan Nacional sobre el SIDA.

A lo largo de la lectura de los artículos se podrá observar como sus autoras/es han aplicado la perspectiva de género en las investigaciones o sus análisis, mediante la adopción de alguno de los tres niveles de observación establecidos a partir de los sistemas de género; los cuales reflejan los procesos que subyacen bajo las desigualdades sociales basadas en las diferencias socialmente construidas en función del sexo. Desde los niveles de observación las autoras y autores de los artículos han caracterizado al género como un componente de la identidad personal, como base de los valores normativos de la sociedad, y/o como un principio organizador de la estructura social (posición social de las mujeres y los hombres).

Para orientar la lectura quiero recordar, que el género como componente de la identidad individual, hace referencia al sentido que las personas tienen de sí mismas frente a la identidad social, que son las categorizaciones de las personas efectuadas por los otros. La identidad propia es una auto-percepción que puede estar más o menos cerca del modelo social de referencia. En este sentido, por otra parte, se entiende que el género está en la base de los valores normativos de la sociedad; y que la identidad social de género se constituye a partir de los patrones sociales asociados a características aparentes, corpóreas y visibles proyectadas sobre los grupos sociales, en concreto, el de las mujeres y de los hombres. Responde a lo que se considera, de forma estereotipada, lo femenino y lo masculino en una sociedad. Y por otra parte, el género se puede entender también como principio organizador de la estructura social, mediante el cual, las actividades de mujeres y hombres en una sociedad están segregadas en función de su sexo. En este nivel de observación se incluyen los estudios sobre la posición de las mujeres y hombres en las sociedades, el acceso a los recursos y la distribución de los mismos. Se incluyen los análisis que consideran al género un criterio de estratificación social, como los relacionados con el trabajo reproductivo (cuidados y doméstico) y remunerado, la doble jornada o la segregación del mercado laboral. Destacan tres escenarios en los que existe un reparto de tareas por sexo, mediante los que se pueden observar los efectos de la división sexual del trabajo: vida familiar-domésticapersonal o privada, vida profesional-laboral o pública, e interacción de ambas vidas, con sus implicaciones en la distribución del tiempo y la sobrecarga de trabajo.

Este número consta de cuatro partes. La primera parte ( Entrevista con dos Salubristas: Desarrollo de las mujeres y cuestiones de género en su historia de vida») tiene por objetivo recordar hitos, situaciones, barreras y evolución 
de la emancipación de las mujeres españolas en nuestra historia reciente y que tanto han influido en la salud de las mismas, así como también, sin duda, en la salud de los hombres españoles. Es a través del transcurso de las vidas de los protagonistas de esta sección, Lucía Mazarrasa Alvear y Carlos Álvarez-Dardet Díaz, que aprendemos a apreciar cómo ha afectado, en tanto que individuos y personas, la complejidad de los valores sociales, una vez transformados en toma de decisiones y acciones por parte de familiares, compañeros de estudios y trabajo, y políticos. Agradezco las lúcidas e inteligentes respuestas y reflexiones a mis preguntas. Hace años supe que una Catedrática de Universidad de Medicina Preventiva y Salud Pública aún en activo, no pudo serlo hasta que se eliminó una normativa por la que para alcanzar este puesto era preciso ser militar. Afortunadamente, la democracia ha ido allanando el camino hacia la igualdad, por el cual transitan profesionales de la salud pública como Carlos Álvarez-Dardet y Lucía Mazarrasa, que van por delante desbrozando los márgenes y apartando las piedras y obstáculos que aún dificultan la igualdad de género y sus consecuencias en la salud pública. He admirado siempre su capacidad profesional, y especialmente su compromiso social y activismo político.

La segunda parte está compuesta por «artículos originales» que aportan nuevos datos y conocimiento sobre problemas de salud pública de gran interés en la actualidad como la violencia de género, el SIDA, el aborto, o el tabaco; así como otros centrados en temas clásicos vistos por una mirada nueva acerca de la maternidad, la menopausia, o sobre los efectos de la representación social de las mamas en la prevención de cáncer mamario. También se incluyen artículos centrados en el análisis de género de herramientas de uso institucional, como los planes directores de salud, la encuesta nacional de salud, e indicadores para realizar un perfil de salud de las mujeres. Son artículos operativos, de los que se puede aprender método para la aplicación de la perspectiva de género. Este apartado contiene un artículo sobre el desarrollo profesional en una profesión sanitaria imprescindible, la enfermería; el cual tiene su complemento en el siguiente apartado, con uno de los ensayos sobre un artículo acerca del desarrollo profesional en medicina.

Una serie de ensayos que hacen referencia a artículos, que podríamos decir son de lectura obligada para aprender sobre la perspectiva y el análisis de género, componen La tercera parte, denominada «Las expertas recomiendan lecturas sobre ...» En efecto, expertas de alguna rama de la salud y la salud pública; así como en la aplicación de la perspectiva y el análisis de género, nos ilustran en cómo hacerlo, mediante un ensayo breve en el que comentan un artículo o libro que han seleccionado, y que recomiendan leer por considerarlo relevante para tal fin. Se incluyen análisis y reflexiones sobre la relación 
entre características demográficas como el estado civil y/o de pareja y su relación con la salud de los y las trabajadores(as), la morbilidad diferencial, el desarrollo profesional dentro de medicina, y de cómo construir y ayudar a la promoción de la salud a través de la perspectiva de género. Además de aprender con la lectura del propio artículo referido, quienes se interesan por cómo aplicar el análisis de género, pueden aprender con las razones y argumentos utilizados por las expertas.

Dada la aspiración de que este número de la revista FEMINISMO/S llegue y su uso sea de utilidad a las y los jóvenes investigadoras/es, se ha incluido la cuarta parte cuyo objetivo es darles la voz. Para ello, con el mismo formato de la tercera parte, tres jóvenes investigadoras en salud pública han escrito con maestría un ensayo en el que cada una nos propone la lectura de un artículo del que han aprendido cómo aplicar la perspectiva de género o hacer análisis de género.

Para acabar. En el número 18 de la revista FEMINISMO/S se encuentran múltiples elementos de ayuda para aprender qué es esto de la perspectiva y el análisis de género, y su aplicación concreta en el ámbito de la salud pública. Parte del conocimiento ha sido producido, por ser objeto de interés de la historia y parte es objeto de interés científico actual: la mujer como objeto del conocimiento científico, la mujer como agente de cuidados de salud, las profesiones y las mujeres. Ambos contienen útiles en la innovación científica. Los textos incluidos aquí se ubican en algunas de las corrientes epistemológicas feministas donde conceptos y métodos se dan la mano; así que no está de más recordar que debemos investigar ateniéndonos a algunas de estas corrientes epistemológicas feministas existentes; tal que las principales: el empirismo feminista, el punto de vista o de partida feminista (feminism standpoint), y el postmodernismo feminista. 